\title{
Massive MIMO Channel Models: A Survey
}

\author{
Kan Zheng, ${ }^{1}$ Suling Ou, ${ }^{1}$ and Xuefeng Yin ${ }^{2}$ \\ ${ }^{1}$ Wireless Signal Processing and Network Lab, Key Laboratory of Universal Wireless Communication, Ministry of Education, \\ Beijing University of Posts and Telecommunications (BUPT), P.O. Box 93, No. 10, Xi Tu Cheng Road, Haidian District, \\ Beijing 100876, China \\ ${ }^{2}$ Department of Electronics Science and Technology, Tongji University, Shanghai, China
}

Correspondence should be addressed to Kan Zheng; zkan@bupt.edu.cn

Received 25 January 2014; Accepted 28 March 2014; Published 16 June 2014

Academic Editor: Periklis Chatzimisios

Copyright (C) 2014 Kan Zheng et al. This is an open access article distributed under the Creative Commons Attribution License, which permits unrestricted use, distribution, and reproduction in any medium, provided the original work is properly cited.

The exponential traffic growth of wireless communication networks gives rise to both the insufficient network capacity and excessive carbon emissions. Massive multiple-input multiple-output (MIMO) can improve the spectrum efficiency (SE) together with the energy efficiency (EE) and has been regarded as a promising technique for the next generation wireless communication networks. Channel model reflects the propagation characteristics of signals in radio environments and is very essential for evaluating the performances of wireless communication systems. The purpose of this paper is to investigate the state of the art in channel models of massive MIMO. First, the antenna array configurations are presented and classified, which directly affect the channel models and system performance. Then, measurement results are given in order to reflect the main properties of massive MIMO channels. Based on these properties, the channel models of massive MIMO are studied with different antenna array configurations, which can be used for both theoretical analysis and practical evaluation.

\section{Introduction}

The requirements for high rate of wireless communication networks grow exponentially with the applications of smart terminals. Thus, the capacity of the networks has to be increased in order to guarantee the quality of service (QoS) of mobile applications. Improving the spectrum efficiency (SE) is one of the feasible ways to achieve the better network capacity. Besides it, with the excessive power consumption of wireless communication networks, both the carbon emissions and operator expenditure increase every year $[1,2]$. Therefore, green communication has gained more attention in the academic and industrial fields, and the energy efficiency (EE) has been regarded as another vital metric for evaluating the new technique as well as the spectrum efficiency [3-5].

Multiple-input multiple-output (MIMO) technique has attracted much attention in wireless communications for more than ten years because it can offer significant increases in data throughput and link reliability without extra bandwidth or boosting transmission power. Nowadays, MIMO together with orthogonal frequency division multiplexing (OFDM) has been accepted as key techniques in the third generation (3G) long-term evolution (LTE) cellular networks and its advancement (Adv.). The evolved Node B (eNB) equipped with multiple antennas communicates with several types of user equipment (UE); at the same time frequency resources, named as multiuser MIMO (MU MIMO), can improve the spectrum efficiency, link reliability, and system energy efficiency $[6,7]$. In order to scale up these benefits, massive MIMO, also named as large-scale antenna system, very large MIMO, or hyper MIMO, has been first put forward in 2010 [8]. In massive MIMO system, hundreds of antennas are used to serve tens of UEs simultaneously. Theoretical and measurement results indicate that massive MIMO can significantly improve the spectrum efficiency and simultaneously reduce the radiated power $[6,9]$. All the features of massive MIMO are shortly summarized in Table 1. 
TABLE 1: Features of massive MIMO.

\begin{tabular}{|c|c|c|}
\hline & Feature & Main reason \\
\hline \multirow{7}{*}{ Advantages } & High spectrum efficiency $[6,9]$ & Large multiplexing gain and array gain \\
\hline & High energy efficiency $[6,9]$ & Radiated energy can be concentrated on UE \\
\hline & High reliability [9] & Large diversity gain \\
\hline & Efficient linear precoder/detector [9] & $\begin{array}{l}\text { Favorable propagation condition for i.i.d. Rayleigh } \\
\text { channel }\end{array}$ \\
\hline & $\begin{array}{l}\text { Weak interuser interference and } \\
\text { enhanced physical security [6] }\end{array}$ & Orthogonal UE channels and extremely narrow beam \\
\hline & Simple scheduling scheme [16] & $\begin{array}{l}\text { Channel harden phenomenon averages out the fast } \\
\text { fading }\end{array}$ \\
\hline & Robust to individual element failure [6] & Large number of antenna array elements \\
\hline \multirow{4}{*}{ Disadvantages } & Pilot contamination $[8]$ & $\begin{array}{l}\text { Limited orthogonal pilots as of bounded coherent } \\
\text { interval and bandwidth }\end{array}$ \\
\hline & High signal processing complexity [9] & Large number of antennas and multiplexing UE \\
\hline & Sensitive to beam alignment [6] & $\begin{array}{l}\text { Extremely narrow beam is sensitive to UE moving or } \\
\text { antenna array swaying }\end{array}$ \\
\hline & Poor broadcast channel [6] & Be blind to UE positions \\
\hline
\end{tabular}

In Release 11 (R11) specified by the 3rd generation partnership project (3GPP), the MIMO technique can only radiate the beam in horizontal dimension as of the fixed down tilt of antenna array. In order to well exploit the vertical angular resolution of signal propagation, different kinds of the antenna array configurations, such as the rectangular, spherical, and cylindrical antenna array deployments, have been studied in 3GPP [13-15]. With these MIMO antenna array deployments, the eNB can adaptively adjust both azimuth and elevation angles of signal propagation. In other words, the radiated MIMO signals can be controllable in the three-dimension $(3 D)$ space and thus called as $3 D$ MIMO. In order to enhance the system capacity, 3D MIMO should employ more antennas. And, massive MIMO should adopt the rectangular, spherical, or cylindrical antenna array configurations for the practical system considering the space of antennas array. Therefore, 3D MIMO with a large number of antennas can be regarded as one of practical forms of massive MIMO.

Up to now, massive MIMO has been paid much attention to by both academic and industry organizations. There are lots of works done for massive MIMO, such as performance analysis, remedies for alleviating pilot contamination, and channel estimation. The channel model is fundamental for theoretical analysis as well as for performance evaluation of massive MIMO system. Therefore, this paper mainly investigates the state of the art of the channel models for the massive MIMO system. The configuration of antenna array as the critical factor deciding the characteristics of massive MIMO channels is first presented briefly. Based on the given antenna array configurations, the current measured activities of massive MIMO channels are studied for working out the main properties of massive MIMO channels, which are the basis for the precise channel models. With the new properties, two kinds of channel models, namely, correlation-based stochastic models (CBSMs) and geometry-based stochastic models (GBSMs), are presented for the massive MIMO in consideration of both the theoretical analysis and realistic system evaluation.

The remainder of this paper is organized as follows. Section 2 discusses antenna array deployments of massive MIMO. In Section 3, measurement results of massive MIMO channel are mainly investigated. In Section 4, both the CBSMs and GBSMs of massive MIMO are studied. Finally, we conclude the paper in Section 5.

\section{Antenna Array Configuration}

The antenna array structures roughly undergo three phases with the development of technique in manufacturing. In the traditional passive antenna array, the radiofrequency circuit is usually connected to the physical antennas through the radiofrequency cable. Subsequently, in order to reduce the loss induced by the radiofrequency cable and save the cost of installation and maintenance, the remote radio unit (RRU) separated from the baseband unit (BBU) has been widely adopted [21]. The digital baseband signals generated from the BBU are sent to the RRUs through the optical fiber. The radiofrequency circuit is as close as possible to the physical antennas. Furthermore, active antenna array system without radiofrequency cable may be made, which is helpful to the realization of the massive antenna array [22]. In active antenna array, both the radiofrequency circuits and the antenna elements are integrated into one circuit board, which is an important milestone for the development of antenna array.

As shown in Figure 1, there are several typical antenna array configurations for massive MIMO systems, that is, linear antenna array, spherical antenna array, cylindrical antenna array, rectangular antenna array, and distributed antenna array [6]. The linear array is an example of onedimension (1D) antenna array, which generally propagates 


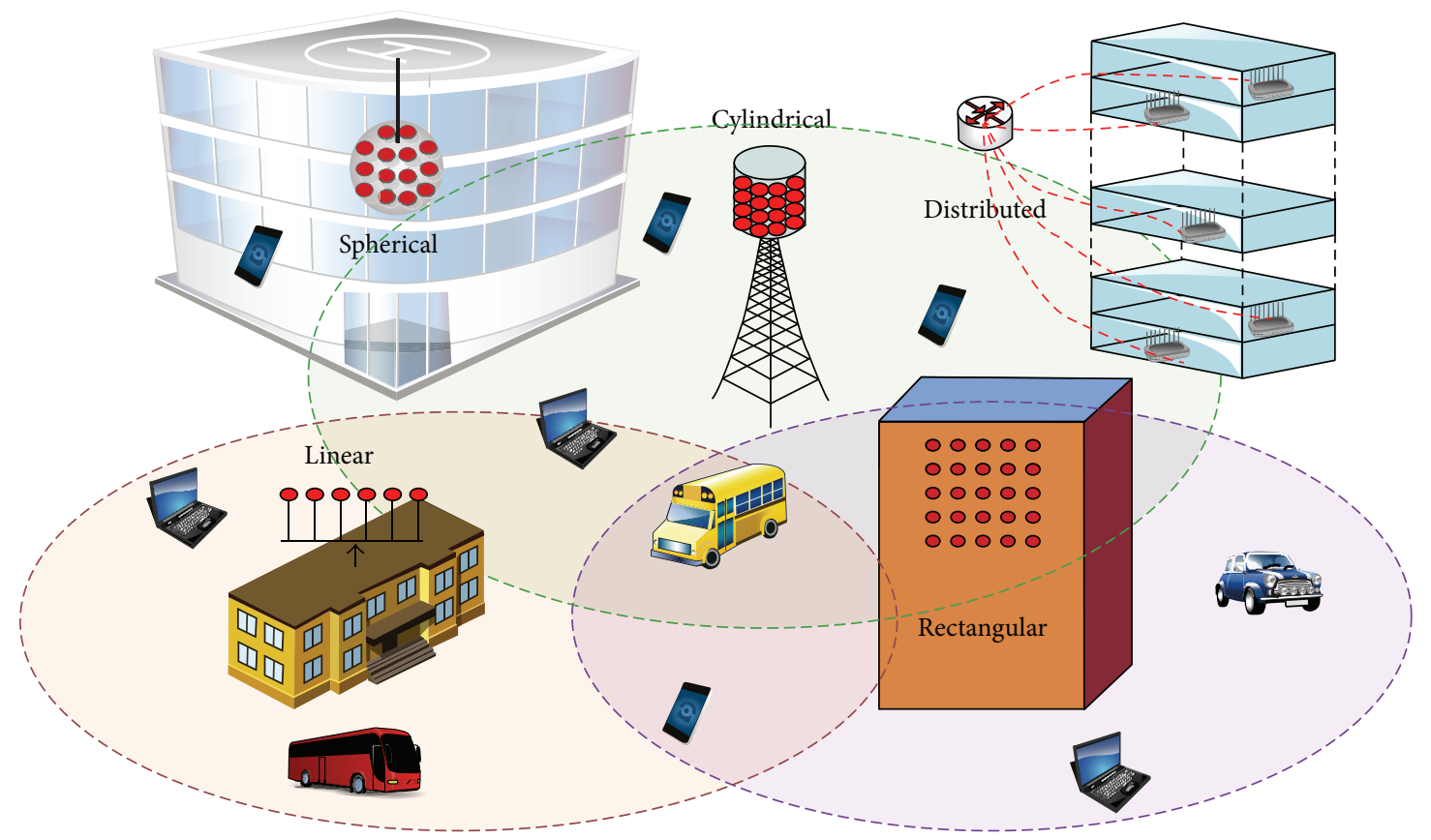

FIgURE 1: Antenna array configuration [6].

signals on the two-dimension (2D) plane. It is usually assumed for theoretical analysis and realistic measurements. On the other hand, the spherical antenna array, cylindrical antenna array, and rectangular antenna array are some kind of $2 D$ antenna array, which can radiate the signals into any directions in the $3 D$ space. Considering the size of antenna array for the eNBs and UEs, the spherical, cylindrical, and rectangular antenna array configurations are more likely to be used in practical systems. Moreover, distributed antenna array is mainly used for either indoor coverage enhancement or outdoor cooperation.

The configurations of antenna array directly affect the channel properties, furthering the performance of massive MIMO system. Taking the antenna space as an example, it decides the mutual coupling and correlation matrix, furthering the capacity of massive MIMO. However, the spaces between adjacent antennas in current antenna array are usually set as equal. Therefore, the study of the antenna array configuration on a constraint or unconstraint area is valuable for the development of massive MIMO, such as the design of sparse antenna array.

\section{Channel Measurement}

Lots of channel measurements have been carried out in order to discriminate the main properties of massive MIMO channel. Typical measurements are summarized in Table 2 for different antenna array configurations under a given scenario. These measurements mainly focus on the impacts of antenna numbers on the small-scale propagation characteristics. When the linear antenna array is employed at the eNB, the nonstationary phenomenon and near-field effect are studied as the main properties for the realistic channel model [17, 23]. However, further measurements should be implemented to validate the two properties for the spherical, cylindrical, and rectangular antenna array configurations. We will discuss the properties derived from channel measurements in detail as follows.

The channel characteristics, such as channel gain, $K$ factor, and angular power spectrum (APS), are measured with a bandwidth of $50 \mathrm{MHz}$, when the eNB employs a linear 128-antenna array with the antenna space of half a wavelength [17]. As shown in Figure 2, measurement results indicate that the antenna array is beyond the Rayleigh distance as of the large length of linear antenna array. Thus, both the far-field and plane wavefront assumptions in the traditional channel models are inappropriate for the massive MIMO channel. Moreover, different antennas at the eNB may observe different sets of clusters in different time slots, which results in the shadow fading over the linear antenna array, namely, nonstationary phenomenon.

When the eNB employed a 32-antenna rectangular array with half a wavelength between the adjacent antennas, the indoor-to-outdoor channels were measured with a bandwidth of $50 \mathrm{MHz}$ [18]. The correlation between different UE channels in measurements is higher than that in independent identical distributed (i.i.d.) Rayleigh channel, since positions of two UEs are too close to undergo the similar channels. Moreover, the average channel correlations for both measurement and i.i.d. Rayleigh channel decrease with the increasing number of antennas; namely, the UE channels can be decorrelated by the very large antenna array.

Channel measurements for both the cylindrical and linear antenna array with 128-antenna were implemented, when 
TABLE 2: Channel measurement.

\begin{tabular}{llccl}
\hline Scenario & Antenna configuration & Antenna space & Bandwidth/carrier & Measured metric \\
\hline $\begin{array}{l}\text { Outdoor [17] } \\
\text { Indoor-to- }\end{array}$ & 128-antenna linear array & Half wavelength & $50 \mathrm{MHz} / 2.6 \mathrm{GHz}$ & $\begin{array}{l}\text { Channel gain, } K \text {-factor, APS, and eigenvalue } \\
\text { distribution correlation of antennas and users }\end{array}$ \\
$\begin{array}{l}\text { outdoor } \\
{[18]}\end{array}$ & 128-antenna rectangular to & Half wavelength & $50 \mathrm{MHz} / 2.6 \mathrm{GHz}$ & Correlation \\
Outdoor [19] & $\begin{array}{l}\text { Cylindrical and linear array with } \\
\text { 128-antenna }\end{array}$ & Half wavelength & $50 \mathrm{MHz} / 2.6 \mathrm{GHz}$ & Large-scale fading and angular resolution \\
Outdoor [20] & 112-antenna virtual array & - & $20 \mathrm{MHz} / 2.6 \mathrm{GHz}$ & Correlation, inverse condition number \\
\hline
\end{tabular}

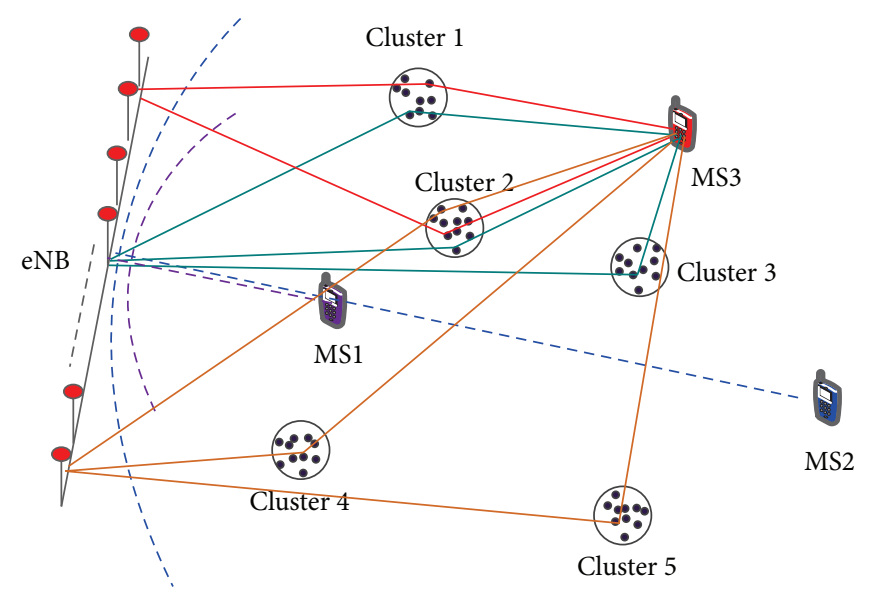

FIGURE 2: Illustration of nonstationary phenomenon and near-field effect [10].

the space of adjacent antennas is half a wavelength and the bandwidth is $50 \mathrm{MHz}$ [19]. Similar to [17], the nonstationary phenomenon can be observed over the linear antenna array. Meanwhile, large power variation can also be experienced over the cylindrical antenna array as of both the polarization and directional pattern. Moreover, the linear antenna array has superior angular resolution but only in azimuth. The cylindrical antenna array has lower angular resolution, yet it can resolve in both azimuth and elevation, which may be an advantage for some scenarios, such as building coverage.

A scalable virtual antenna array with 112-antenna was employed at the eNB in order to investigate the channel characteristics for massive MIMO in [20]. Both the line of sight (LoS) and non-line of sight (NLoS) scenarios were studied with a bandwidth of $20 \mathrm{MHz}$. The correlation and inverse condition number were measured to evaluate the orthogonality of two-channel vectors and multiple channel vectors, respectively. The difference of inverse condition numbers for measured channel and i.i.d. Rayleigh channel becomes larger with more multiple UE and/or deploying more antennas. Deploying more antennas will make the channels of multiple UE more orthogonal. However, there is less improvement when the number of antennas exceeds a given number, which mainly relies on the propagation environment and UE positions.

The measurements for the characteristics of elevation angles are extensive for $3 D$ MIMO system recently $[11,24]$. The main measurement results of rectangle antenna array have been concluded in 3GPP [11]. The channel model of rectangle antenna array comprising these characteristics is also established, which will be studied in the next section.

\section{Channel Model}

Two kinds of channel models, namely, correlation-based stochastic models (CBSMs) and geometry-based stochastic models (GBSMs), are widely used to evaluate the performances of the wireless communication systems. The complexity of the former is lower and is mainly used for analyzing the theoretical performance of MIMO systems. However, the accuracy is limited for the realistic MIMO system, and it is difficult to model wireless channels considering the nonstationary phenomenon and spherical wave effects. In contrast, the latter can accurately reflect the realistic channel properties and is more suitable for massive MIMO channel even with the higher computation complexity. The current channel models have been summarized in Table 3. This section discusses the CBSMs for theoretical analysis and GBSMs for the realistic performance evaluation according to the categories in Table 3.

4.1. CBSM. The CBSMs are mainly used as the theoretical model to evaluate the performance of massive MIMO system. The current analysis generally assumes that the UEs employ single antenna in consideration of the complexity and spaces 
TABLE 3: Channel models of massive MIMO.

\begin{tabular}{lll}
\hline Modeling method & Category & Property \\
\hline CBSM & $\begin{array}{l}\text { i.i.d. Rayleigh channel model } \\
\text { Correlation channel model }\end{array}$ & $\begin{array}{l}\text { Elements of fast fading are i.i.d. complex Gaussian variables } \\
\text { Contain correlation between transmit antennas or/and receive } \\
\text { antennas } \\
\text { Consider antenna impedance, load impedance, and mutual } \\
\text { impedance }\end{array}$ \\
\hline GBSM & $2 D$ channel model & $\begin{array}{l}\text { Propagate beam on } 2 D \text { plane, such as linear array } \\
\text { Propagate beam on } 3 D \text { plane, such as spherical, rectangular, and } \\
\text { cylindrical array }\end{array}$ \\
\hline
\end{tabular}

for the terminal equipment with low carrier frequency. Therefore, the following CBSMs are discussed when the UE employs single antenna, which are easy to be extended to the cases that the UE with multiple antennas works at the millimeter wave. Moreover, massive MIMO is more likely to be used in time division duplex (TDD) system at present, since the downlink channel state information (CSI) can be acquired from the uplink due to TDD reciprocity feature. Therefore, only the uplink channel is taken as an example for study in this section.

Let us consider an uplink MIMO system that $K$ singleantenna UE transmits signals to the eNB equipped with $N$ antennas simultaneously. When the NLoS channel is assumed, the channel model can be generalized by

$$
\mathbf{G}=\mathbf{H D}^{1 / 2}
$$

where $\mathbf{D}=\operatorname{diag}\left\{\beta_{1}, \beta_{2}, \ldots, \beta_{K}\right\}$ is the large-scale propagation matrix and $\beta_{k}=\phi d_{k}^{-\alpha} \xi_{k}$. $\phi$ is a constant related to the antenna gain and carrier frequency, $d_{k}$ is the distance between the eNB and the $k$ th UE, $\alpha$ is the path loss exponent, and $\xi_{k}$ is the log-normal shadow fading with $10 \log _{10} \xi_{k} \sim \mathcal{N}\left(0, \sigma_{\mathrm{sh}}^{2}\right)$. $\mathbf{H} \in \mathscr{C}^{N \times K}$ is the fast fading matrix.

According to the fast fading matrix, the CBSMs can be further simplified into three kinds of channels, namely, i.i.d. Rayleigh fading model, correlation channel model, and mutual coupling channel model. We discuss these three kinds of models as follows in detail.

4.1.1. i.i.d. Rayleigh Channel Model. The i.i.d. Rayleigh fading channel model is widely adopted for the theoretical analysis of the massive MIMO system, which assumes there is no correlation and mutual coupling between transmit antennas or receiver antennas. The elements of the fast fading matrix $\mathbf{H}=\left[\mathbf{h}_{1}, \mathbf{h}_{2}, \ldots, \mathbf{h}_{K}\right]$ are i.i.d. Gaussian random variables; namely, $h_{n, k} \sim \mathscr{C} \mathcal{N}(0,1)(n=1,2, \ldots, N ; k=1,2, \ldots, K)$.

The favorable propagation is the most important property of the i.i.d. Rayleigh fading channel in the massive MIMO system [8]; namely,

$$
\frac{1}{N} \mathbf{G}^{H} \mathbf{G} \approx \mathbf{D}, \quad N \gg K
$$

According to the large number of laws, we can find one characteristic of the favorable propagation is the orthogonality of different UE channels:

$$
\frac{1}{N} \mathbf{h}_{i}^{H} \mathbf{h}_{j} \approx \begin{cases}0, & i \neq j \\ 1, & i=j .\end{cases}
$$

Another is the channel harden phenomenon; namely, the Euclidean norm of each UE channel approximates to the large-scale fading factor [9]:

$$
\frac{1}{N}\left\|\mathbf{g}_{k}\right\|^{2} \approx \beta_{k}, \quad k=1,2, \ldots, K .
$$

The favorable propagation can not only better the performance but also simplify the algorithm design of massive MIMO system. The orthogonality can alleviate the interuser/intercell interference, which is helpful to improve the system capacity. Channel harden phenomenon can mitigate the impact of fast fading on the scheduling gain, which simplifies the complexity of scheduling scheme [16].

4.1.2. Correlation Channel Model. In order to reflect the antenna correlation caused by the insufficient antenna space and the scattering environments, the correlation channel model is established to evaluate the performance of the massive MIMO system [25]. The fast fading channel vector of each one of UE can be formed by the correlation matrix multiplied by standard complex Gaussian vector; namely,

$$
\mathbf{h}_{k}=\mathbf{R}_{k} \mathbf{v}_{k}, \quad k=1,2, \ldots, K,
$$

where the steering matrix $\mathbf{R}_{k} \in \mathscr{C}^{N \times D_{k}}$ contains $D_{k}$ steering vectors with different angles of arrival (AoAs) for the $k$ th UE and $\mathbf{v}_{k} \sim \mathscr{C} \mathscr{N}\left(\mathbf{0}, \mathbf{I}_{D_{k}}\right)$.

When the linear antenna array is assumed at the eNB, the steering matrix $\mathbf{R}_{k}$ can be written as

$$
\mathbf{R}_{k}=\frac{1}{D_{k}}\left[\mathbf{a}\left(\theta_{k, 1}\right), \mathbf{a}\left(\theta_{k, 2}\right), \ldots, \mathbf{a}\left(\theta_{k, D_{k}}\right)\right],
$$

where $\theta_{k, i}$ is the $i$ th AoA of $k$ th UE and the steering vector $\mathbf{a}\left(\theta_{k, i}\right) \in \mathscr{C}^{N \times 1}$ is given as

$$
\mathbf{a}\left(\theta_{k, i}\right)=\left[1, e^{(j 2 \pi d / \lambda) \sin \theta_{k, i}}, \ldots, e^{(j 2 \pi(N-1) d / \lambda) \sin \theta_{k, i}}\right]^{T},
$$

where $d$ is the distance between the adjacent antennas and $\lambda$ is the carrier wavelength. 
For the rectangle antenna array, the steering vector can be attained by the steering matrix; namely,

$$
\begin{aligned}
& \mathbf{a}\left(\theta_{k, i}, \phi_{k, i}\right) \\
& =\operatorname{vec}\left\{\left[1, e^{(j 2 \pi d / \lambda) \sin \theta_{k, i}}, \ldots, e^{(j 2 \pi(N-1) d / \lambda) \sin \theta_{k, i}}\right]^{T}\right. \\
& \left.\otimes\left[1, e^{(j 2 \pi d / \lambda) \sin \phi_{k, i}}, \ldots, e^{(j 2 \pi(N-1) d / \lambda) \sin \phi_{k, i}}\right]\right\},
\end{aligned}
$$

where $\theta_{k, i}$ and $\phi_{k, i}$ represent azimuth of arrival (AoA) and elevation of arrival (EoA), respectively. $\operatorname{vec}\{\cdot\}$ denotes vectorization of matrix. Note that AoA denotes the abbreviation of angle of arrival or azimuth of arrival for linear antenna array or rectangle antenna array, respectively.

This correlation channel model introduces the AoAs, which can be utilized to distinguish the UE and improve the accuracy of channel estimation [26]. When the UE is located at different orientations, the UE channels can almost be separated by angle information, thereby alleviating the pilot contamination. It is also helpful to analyze the interuser or intercell interference and develop UEs scheduling or cell cooperation to alleviate the interference.

4.1.3. Mutual Coupling Channel Model. Since the number of antennas increases sharply in the massive MIMO system, the mutual impedance has to be considered as of the limited space for antenna array. Moreover, the load impedance and antenna impedance should also be characterized in order to reflect the realistic channel model.

The channel vector of the $k$ th UE can be written as follows in consideration of both the impedances and correlation [27]:

$$
\mathbf{h}_{k}=\mathbf{Z R}_{k} \mathbf{v}_{k}, \quad k=1,2, \ldots, K,
$$

where $\mathbf{Z} \in \mathscr{C}^{N \times N}$ represents the mutual coupling matrix, $\mathbf{R}_{k} \in$ $\mathscr{C}^{N \times D_{k}}$ denotes the steering matrix containing $D_{k}$ steering vectors of the receiver antenna array, and $\mathbf{v}_{k} \sim \mathscr{C} \mathscr{N}\left(\mathbf{0}, \mathbf{I}_{D_{k}}\right)$. According to $[27,28]$, the mutual coupling matrix can be expressed as

$$
\mathbf{Z}=\left(Z_{A}+Z_{L}\right)\left(\boldsymbol{\Gamma}+Z_{L} \mathbf{I}\right)^{-1}
$$

with

$$
\boldsymbol{\Gamma}=\left[\begin{array}{ccccc}
Z_{A} & Z_{M} & 0 & \cdots & 0 \\
Z_{M} & Z_{A} & Z_{M} & \cdots & 0 \\
0 & Z_{M} & Z_{A} & \cdots & 0 \\
\vdots & \vdots & \ddots & \ddots & \vdots \\
0 & 0 & \cdots & Z_{M} & Z_{A}
\end{array}\right]
$$

where $Z_{A}, Z_{L}$, and $Z_{M}$ represent the antenna impedance, load impedance, and mutual impedance, respectively. Here, the mutual impedances are only considered between adjacent antennas, which can be seen from the nonzero $Z_{M}$. The further discussion about the mutual coupling based on the electromagnetic analysis can be found in $[29,30]$. Therefore, the transmit correlation matrix can be written as follows [27]:

$$
\boldsymbol{\Sigma}_{N}=E\left[\mathbf{H} \mathbf{H}^{H}\right]=K \mathbf{Z} \mathbf{R} \mathbf{R}^{H} \mathbf{Z}^{H},
$$

where $\mathbf{R}=\left[\mathbf{R}_{1}, \mathbf{R}_{2}, \ldots, \mathbf{R}_{K}\right]$. As the UE is geometrically distributed, the received correlation matrix can be assumed as $\mathbf{I}_{K}$. Then, we can get $\mathbf{H} \sim \mathscr{C} \mathscr{N}\left(0, \boldsymbol{\Sigma}_{N} \otimes \mathbf{I}_{K}\right)$.

Mutual coupling channel model is more practical for massive MIMO. It is helpful to analyze the effects of antenna space on the performance of massive MIMO, which is significant for designing the antenna array configuration, especially the sparse antenna array.

4.2. GBSM. GBSMs are mainly used for evaluating the performance of practical wireless communication systems, which accurately comprise the channel properties. Considering the elevation or not, the GBSMs of massive MIMO can be classified into two kinds, namely, $2 D$ and $3 D$ channel models. When the linear antenna array is employed at the eNB, the tilt angle is fixed and the $2 D$ channel model is precisely enough for evaluating the performance. However, if the spherical, cylindrical, or rectangular antenna array configurations are adopted at the eNB, the $3 D$ channel model in consideration of both elevation and azimuth should be established for evaluation.

4.2.1. 2D Channel Model. Employing the linear antenna array, the nonstationary phenomenon and spherical wavefront have been discriminated as the main properties for the massive MIMO channel through channel measurements $[17,19]$. The properties are helpful to decorrelate the channels for different type of UE, thereby providing a favorable channel [17]. However, they raise the difficulty to establish the channel model for the massive MIMO system. Therefore, the realistic channel model comprising the properties is still on the way at present [10].

Similar to $[17,19]$, the nonstationary phenomenon is also observed through the measurements for the linear 128antenna array in a semiurban area [31]. In order to test the performance of proposed algorithms for the more practical massive MIMO system, much work has been done on the channel modeling of massive MIMO reflecting this nonstationary phenomenon. Therefore, a cluster-based channel model comprising the nonstationary phenomenon has been established, which can be seen as the extension of the COST 2100 channel model.

In consideration of both the nonstationary phenomenon and spherical waveform effect, an elliptical GBSM was given for massive MIMO with linear antenna array [23, 32, 33]. First, clusters on lots of confocal ellipses with different major axis represent different resolvable delays, which make the GBSM possesses the spherical wavefront property. Then, the birth-death process of clusters is introduced to describe the nonstationary property. Numerical results of the established channel demonstrate that the phases responses of the linear antenna array are no longer linear and the AoAs of the antenna array shift gradually, which coincides with the measurement results in the realistic environments.

4.2.2. 3D Channel Model. The 3D channel models have been investigated in the academic for several years already, such as $[34,35]$. Meanwhile, several projects have been established 
especially for the $3 D$ channel model, such as WINNER + project [36]. However, most of these models mainly depend on the literature survey rather than on realistic measurements [24]. Thus, they are considered insufficient or obviously questionable for the realistic channel properties, such as the cross-correlation matrix of large-scale parameters. Therefore, the existing $3 D$ channel models need to be improved through realistic measurements and further used to evaluate the realistic scenarios.

In WINNER + projects, the main procedures of $3 D$ MIMO channel model have been described in detail [11, 12, 37], as shown in Figure 3. The main parameters of $3 D$ channel model contain shadow fading (SF), delay spread (DS), $K$ factor, AoA, azimuth of departure (AoD), EoA, and elevation of departure (EoD), especially the EoA and EoD. The crosscorrelation matrix has been expanded to 7-dimension from 5 -dimension in the $2 D$ channel model. In the stage of 3GPP Release 12 (R12), three scenarios have been defined, namely, urban microcell with high UE density (3D-UMi), urban macrocell with high UE density (3D-UMa), and urban macrocell with one high-rise per sector and $300 \mathrm{~m}$ intersite distance (ISD) (3D-UMa-H). Based on the procedure in Figure 3 , the $3 D$ channel model of rectangle antenna array has been given based on measurements. The related parameters, their distributions, and the cross-correlation matrix for the $3 D$ channel model have been concluded in the technical specification [11]. Next, we will discuss the modeling procedure briefly according to [11, 12, 36, 37].

The large-scale propagation of the $3 D$ MIMO channel consists of both the path loss and shadow fading. The path loss model has to be classified into outdoor and outdoorto-indoor (O2I) models in consideration of the different propagation environments [11]. The illustration of path loss for both outdoor and O2I can be simply depicted in Figure 4. In the outdoor scenarios, the path loss is the function of the carrier frequency $f_{0}$, distance $d_{3 D}$ between the eNB and the $\mathrm{UE}$, break point distance $d_{\mathrm{bp}}$, the height of UE $h_{\mathrm{UE}}$, and the height of eNB $h_{\mathrm{eNB}}$; namely,

$$
\mathrm{PL}_{\mathrm{OD}}=f_{\mathrm{OD}}\left(f_{0}, d_{3 D}, d_{\mathrm{bp}}, h_{\mathrm{UE}}, h_{\mathrm{eNB}}\right) \text {. }
$$

As for the O2I scenarios, three items constitute the path loss, namely, basic path loss $\mathrm{PL}_{\mathrm{OD}}$ related to $d_{3 D \text {-out }}$ and $d_{3 D \text {-in }}$ which is similar to the outdoor scenario, the loss through wall $\mathrm{PL}_{\mathrm{TW}}$, and the indoor loss $\mathrm{PL}_{\mathrm{IN}}=0.5 d_{2 D \text {-in }}$; namely,

$$
\mathrm{PL}_{\mathrm{O} 2 \mathrm{I}}=\mathrm{PL}_{\mathrm{OD}}+\mathrm{PL}_{\mathrm{TW}}+\mathrm{PL}_{\mathrm{IN}} \text {. }
$$

The shadow fading $\xi_{n}$ is dependent on the cluster $n$, which follows log-normal distribution; namely,

$$
f_{\xi_{n}}(x)=\frac{\vartheta}{x} \frac{1}{\sqrt{2 \pi \sigma_{\mathrm{sh}, n}^{2}}} \exp \left\{-\frac{(\vartheta \ln x)^{2}}{2 \sigma_{\mathrm{sh}, n}^{2}}\right\}, \quad \vartheta=\frac{10}{\ln 10}
$$

The correlation between adjacent shadow fading values can be characterized as $R(\Delta x)=\exp \left\{-|\Delta x| / d_{\text {cor }}\right\}$, where $d_{\text {cor }}$ is the correlation distance related to the propagation environments.
Generally, the propagation channel contains several clusters $(n=1,2, \ldots)$ with different delay $\tau_{n}$ and power factors. Each cluster contains some rays $(m=1,2, \ldots, M)$ with the same delay. However, in the $3 D$ channel model, the two strongest clusters are spread into three subclusters, with fixed delay offset 0,5 , and $10 \mathrm{~ns}$. The other related delays, angles, and power can be generated by the given distributions and some measured parameters in [11].

When the double polarization array is adopted at the eNB, as shown in Figure 5, the $n$th cluster of the fast fading channel from the $s$ th antenna of the eNB to the $u$ th antenna of the UE in the NLoS scenario can be expressed as follows [11, 12, 24, 36, 37]:

$$
\begin{aligned}
H_{u, s, n}(t)= & \sqrt{P_{n}} \sum_{m=1}^{M}\left[\begin{array}{cc}
F_{r x, u, \theta}\left(\theta_{n, m, \mathrm{EoA}}, \varphi_{n, m, \mathrm{AoA}}\right) \\
F_{r x, u, \varphi}\left(\theta_{n, m, \mathrm{EoA}}, \varphi_{n, m, \mathrm{AoA}}\right)
\end{array}\right]^{T} \\
& \times\left[\begin{array}{cc}
e^{j \Phi_{n, m}^{\theta \theta}} & \sqrt{\kappa_{n, m}^{-1}} e^{j \Phi_{n, m}^{\theta \varphi}} \\
\sqrt{\kappa_{n, m}^{-1}} e^{j \Phi_{n, m}^{\varphi \theta}} & e^{j \Phi_{n, m}^{\varphi \varphi}}
\end{array}\right] \\
& \times\left[\begin{array}{l}
F_{t x, s, \theta}\left(\theta_{n, m, \mathrm{EoD}}, \varphi_{n, m, \mathrm{AoD}}\right) \\
F_{t x, s, \varphi}\left(\theta_{n, m, \mathrm{EoD}}, \varphi_{n, m, \mathrm{AoD}}\right)
\end{array}\right] \\
& \times e^{j 2 \pi\left(\hat{r}_{r x, n, m}^{T} \cdot \bar{d}_{r x, u} / \lambda_{0}+\bar{r}_{t x, n, m}^{T} \cdot \bar{d}_{t x, s} / \lambda_{0}+v_{n, m} t\right)}
\end{aligned}
$$

where $P_{n}$ is the power normalized factor such that the power of all clusters equals one. $\left\{\Phi_{n, m}^{\theta \theta}, \Phi_{n, m}^{\theta \varphi}, \Phi_{n, m}^{\varphi \theta}, \Phi_{n, m}^{\varphi \varphi}\right\}$ are four random initial phases of different polarisation combinations for the $m$ th ray of the $n$th cluster, and $\kappa_{n, m}$ is the cross polarisation power ratio. $F_{r x, u, \theta}$ and $F_{r x, u, \varphi}$ are the patterns of the $u$ th receiver antenna in the direction of the spherical basis vectors $\hat{\theta}$ and $\widehat{\varphi}$, respectively. Similarly, $F_{t x, s, \theta}$ and $F_{t x, s, \varphi}$ are the same for the $s$ th transmit antenna. The spherical unit vector $\widehat{r}_{x, n, m}$ can be given as

$$
\widehat{r}_{\mathrm{x}, n, m}=\left[\begin{array}{c}
\cos \theta_{n, m, \text { Eoy }} \cos \varphi_{n, m, \text { Aoy }} \\
\cos \theta_{n, m, \text { Eoy }} \sin \varphi_{n, m, \text { Aoy }} \\
\sin \theta_{n, m, \text { Eoy }}
\end{array}\right] \text {, }
$$

where " $x=t x, y=A$ " and " $x=r x, y=D$ " represent the spherical unit vectors for receiver and transmitter, respectively. $\bar{d}_{r x, u}$ is the location vector of the $u$ th receiver antenna, $\bar{d}_{t x, s}$ is the location vector of the $s$ th transmit antenna, and $\lambda_{0}$ is the wavelength. Doppler frequency $f_{n, m}=\widehat{r}_{r x, n, m}^{T} \cdot \bar{v} / \lambda_{0}$ with $\bar{v}=v \cdot\left[\cos \theta_{v} \cos \varphi_{v}, \cos \theta_{v} \sin \varphi_{v}, \sin \theta_{v}\right]^{T}$. 


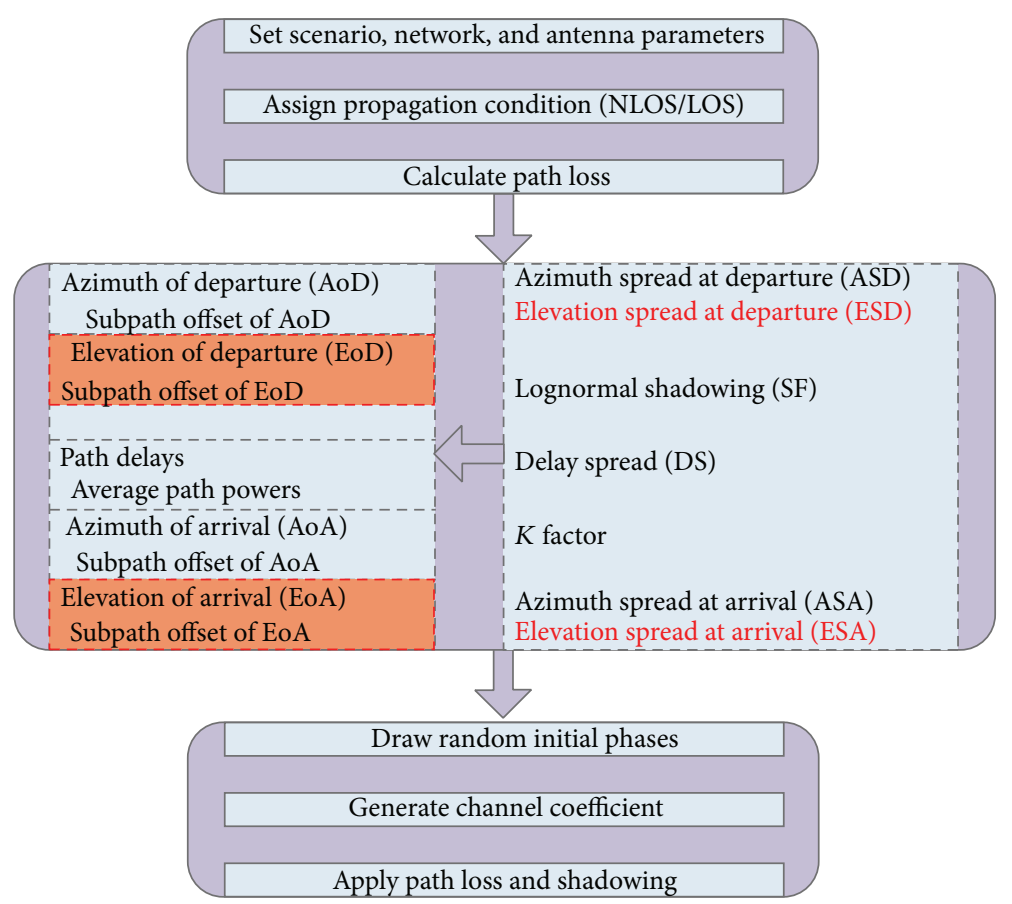

Figure 3: The procedure of $3 D$ channel model $[11,12]$.

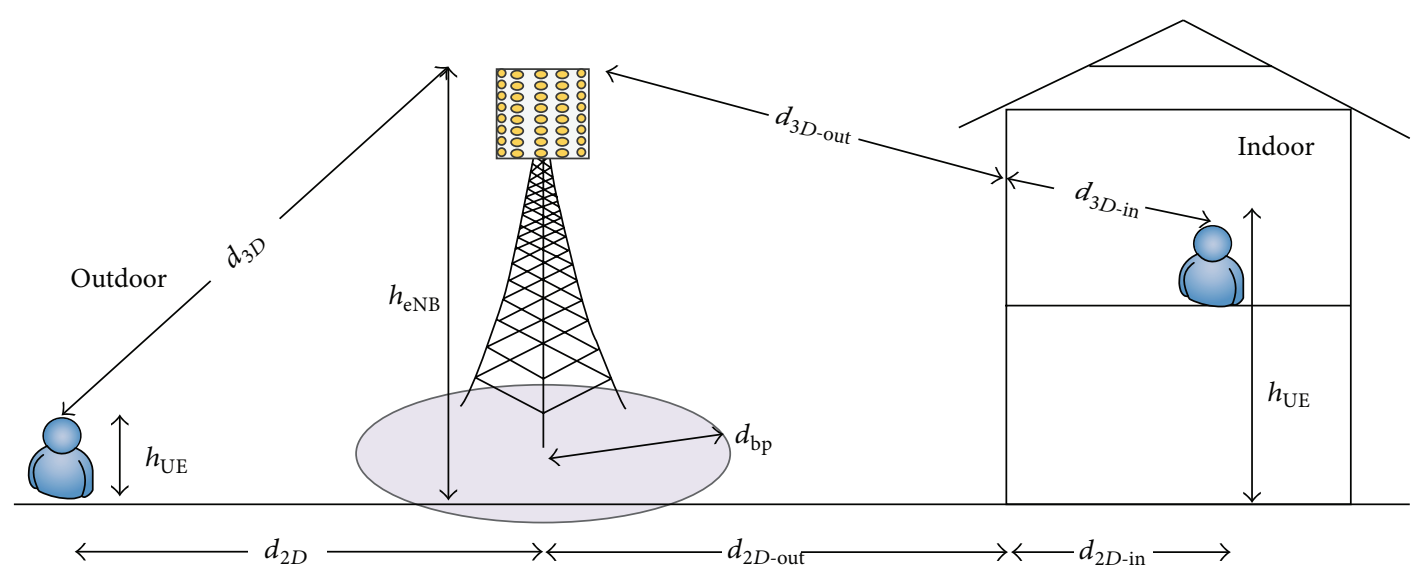

FIGURE 4: Illustration of path loss [11].

As for the LoS scenario, the fast fading with a main path can be written as follows [11, 12, 24, 36, 37]:

$$
\begin{aligned}
& \widetilde{\mathbf{H}}_{u, s, n}(t)=\sqrt{\frac{1}{K_{R}+1}} \mathbf{H}_{u, s, n}(t)+\delta(n-1) \sqrt{\frac{K_{R}}{K_{R}+1}} \\
& \times \sum_{m=1}^{M}\left[\begin{array}{l}
F_{r x, u, \theta}\left(\theta_{\text {LoS,EoA }}, \varphi_{\text {LoS }, \text { AoA }}\right) \\
F_{r x, u, \varphi}\left(\theta_{\text {LoS,EoA }}, \varphi_{\text {LoS }, \text { AoA }}\right)
\end{array}\right]^{T} e^{j \Phi_{\text {LoS }}} \\
& \times\left[\begin{array}{cc}
1 & 0 \\
0 & -1
\end{array}\right]\left[\begin{array}{l}
F_{t x x, \theta, \theta}\left(\theta_{\mathrm{LoS}, \mathrm{EoD}}, \varphi_{\mathrm{LoS}, \mathrm{AoD}}\right) \\
F_{t x, v, \varphi}\left(\theta_{\mathrm{LoS}, \mathrm{EoD}}, \varphi_{\mathrm{LoS}, \mathrm{AoD}}\right)
\end{array}\right]
\end{aligned}
$$

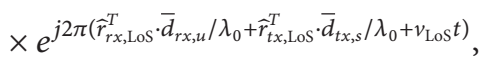

where $\delta(\cdot)$ and $K_{R}$ are the indicative function and Rice factor, respectively.

If only the vertical polarization is adopted in LoS or NLoS scenarios, the $2 \times 2$ polarisation matrix in (16) and (18) can be replaced by $\exp \left(j \Phi_{n, m}\right)$, and only vertically polarization pattern is applied [11, 12].

According to the above procedures of channel modeling, we can generate the realistic channel coefficients to evaluate the three aforementioned scenarios. However, the channel model is not precise for simulations, since the nonstationary phenomenon has not been reflected [19]. Moreover, the channel models of other scenarios and antenna array configurations have to be given in order to extend the applications of massive MIMO. Therefore, the properties and modeling of $3 D$ MIMO channel still need to be further investigated. 


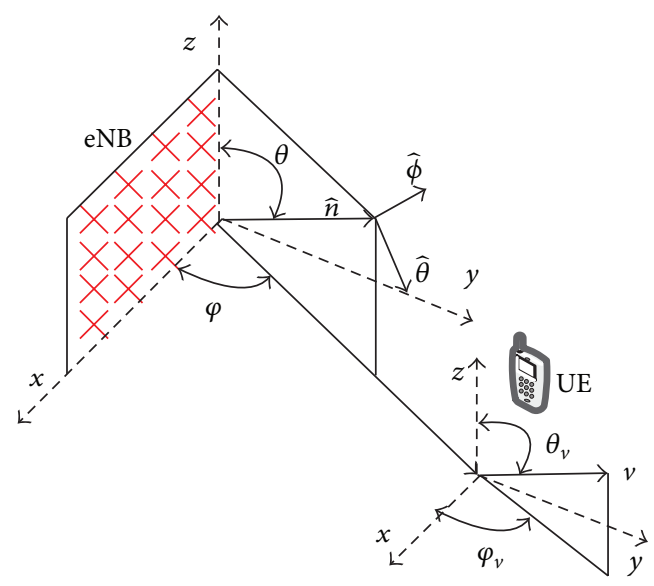

FIGURE 5: Illustration of coordinate [11, 12].

\section{Conclusion}

Massive MIMO has been regarded as one of efficient ways to improve both spectrum efficiency and energy efficiency for the broadband wireless communication systems. As the first step to evaluate the performance of any communication systems, the channel models for massive MIMO are necessary to be investigated, which is the main concern of this paper. According to the ability of array to radiate the signals, the antenna array configurations are classified into $2 D$ and $3 D$ antenna arrays. Generally, the linear antenna array is $2 D$ antenna array, and the rectangle, spherical, and cylindrical antenna array belong to $3 D$ antenna array. From studying the current measurement results of different configurations, it can be found that the linear antenna array gives rise to the nonstationary phenomenon and near-field effect. The $3 D$ antenna array has lower angular resolution, yet it can resolve in both azimuth and elevation. Increasing the number of antennas at eNB can decorrelate different user channels.

At present, CBSMs are mainly used to analyze the theoretical performance of massive MIMO due to its simplification. There are three kinds of simplified CBSMs widely used for different objectives, namely, i.i.d. Rayleigh channel model, correlation channel model, and mutual coupling channel model. The channel model reflecting both the nonstationary phenomenon and spherical wave effect has been established for the linear antenna array based on the cluster model. The improved $3 D$ channel model of rectangle antenna array has been proposed in 3GPP in order to evaluate three kinds of scenarios. However, the channel models of other antenna array configurations and scenarios have to be supplemented, and the nonstationary property for spherical, cylindrical, and rectangular antenna array needs further measurements. Therefore, the channel model of massive MIMO is still an open topic, which needs much more investigation for its improvements in the next steps.

\section{Conflict of Interests}

The authors declare that there is no conflict of interests regarding the publication of this paper.

\section{Acknowledgments}

The work was supported by the China Natural Science Funding (61271183), Program for New Century Excellent Talents in University (NCET-11-0600), National Key Technology R\&D Program of China under Grant 2013ZX03003005, and National High Technology Research and Development Program of China (2014AA01A705).

\section{References}

[1] Z. Hasan, H. Boostanimehr, and V. K. Bhargava, "Green cellular networks: a survey, some research issues and challenges," IEEE Communications Surveys \& Tutorials, vol. 13, no. 4, pp. 524-540, 2011.

[2] D. Feng, C. Jiang, G. Lim, L. J. Cimini Jr., G. Feng, and G. Y. Li, "A survey of energy-efficient wireless communications," IEEE Communications Surveys \& Tutorials, vol. 15, no. 1, pp. 167-178, 2013.

[3] Y. Chen, S. Zhang, S. Xu, and G. Y. Li, "Fundamental trade-offs on green wireless networks," IEEE Communications Magazine, vol. 49 , no. 6 , pp. 30-37, 2011.

[4] G. Y. Li, Z. Xu, C. Xiong et al., "Energy-efficient wireless communications: tutorial, survey, and open issues," IEEE Wireless Communications, vol. 18, no. 6, pp. 28-35, 2011.

[5] G. Auer, V. Giannini, C. Desset et al., "How much energy is needed to run a wireless network?" IEEE Wireless Communications, vol. 18, no. 5, pp. 40-49, 2011.

[6] E. G. Larsson, O. Edfors, F. Tufvesson, and T. L. Marzetta, "Massive MIMO for next generation wireless systems," IEEE Communications Magazine, vol. 52, no. 2, pp. 186-195, 2013.

[7] A. Ghrayeb and T. M. Duman, Coding for MIMO Communication System, John Wiley \& Sons, New York, NY, USA, 2007.

[8] T. Marzetta, "Noncooperative cellular wireless with unlimited numbers of base station antennas," IEEE Wireless Communications, vol. 9, no. 11, pp. 3590-3600, 2010.

[9] F. Rusek, D. Persson, B. K. Lau et al., "Scaling up MIMO: opportunities and challenges with very large arrays," IEEE Signal Processing Magazine, vol. 30, no. 1, pp. 40-60, 2013.

[10] F. Tufvesson, "Very large MIMO systems ICASSP, tutorial-part II: propagation aspects of very large MIMO," in Proceedings of the 37th IEEE International Conference on Acoustics, Speech, and Signal Processing (ICASSP '12), 2012.

[11] "Technical specification group radio access networkstudy on 3D channel model for LTE, (Rel 12)," 3GPP TR- 36.873 V1.1.1, 2013.

[12] Y.-H. Nam, B. Ng, K. Sayana et al., "Full-dimension MIMO (FD-MIMO) for next generation cellular technology," IEEE Communications Magazine, vol. 51, no. 6, pp. 172-179, 2013.

[13] "Requirements, candidate solutions and technology roadmap for lte rel-12 onward," 3GPP RWS-120010, 2012.

[14] “Technologies for Rel-12 and onward," 3GPP RWS- 120021, 2013.

[15] "Views on Rel-12 and onwards for LTE and UMTS," 3GPP RWS120006, 2013. 
[16] B. M. Hochwald, T. L. Marzetta, and V. Tarokh, "Multipleantenna channel hardening and its implications for rate feedback and scheduling," IEEE Transactions on Information Theory, vol. 50, no. 9, pp. 1893-1909, 2004.

[17] S. Payami and F. Tufvesson, "Channel measurements and analysis for very large array systems at $2.6 \mathrm{GHz}$," in Proceedings of the 6th European Conference on Antennas and Propagation (EuCAP '12), pp. 433-437, Prague, Czech Republic, March 2012.

[18] X. Gao, O. Edfors, F. Rusek, and F. Tufvesson, "Linear precoding performance in measured very-large MIMO channels," in Proceedings of the 74th IEEE Vehicular Technology Conference (VTC '11), pp. 1-5, Budapest, Hungary, September 2011.

[19] X. Gao, F. Tufvesson, O. Edfors, and F. Rusek, "Measured propagation characteristics for very-large MIMO at 2.6 GHz," in Proceedings of the 46th IEEE Asilomar Conference on Signals, Systems and Computers (ASILOMAR '12), pp. 295-299, Pacific Grove, Calif, USA, November 2012.

[20] J. Hoydis, C. Hoek, T. Wild, and S. ten Brink, "Channel measurements for large antenna arrays," in Proceedings of the 9th IEEE International Symposium on Wireless Communication Systems (ISWCS '12), pp. 811-815, Paris, France, August 2012.

[21] "C-RAN: the road towards green radio access network," Tech. Rep., China Mobile Research Institute, 2012.

[22] "Active antenna system: utilizing the full potential of radio sources in the spatial domain," Tech. Rep., Huawei, 2012.

[23] C.-X. Wang and S. Wu, "Massive MIMOchannel measurements and modeling: advances and challenges," submitted to IEEE Wireless Communion Magazine.

[24] Z. Zhong, X. Yin, X. Li, and X. Li, "Extension of ITU IMTadvanced channel models for elevation domains and line-ofsight scenarios," in Proceedings of the 78th IEEE Vehicular Technology Conference (VTC '13), pp. 1-5, Las Vegas, Nev, USA, September 2013.

[25] J. Hoydis, S. ten Brink, and M. Debbah, "Massive MIMO in the UL/DL of cellular networks: how many antennas do we need?" IEEE Journal on Selected Areas in Communications, vol. 31, no. 2, pp. 160-171, 2013.

[26] H. Yin, D. Gesbert, M. Filippou, and Y. Liu, "A coordinated approach to channel estimation in large-scale multiple-antenna systems," IEEE Journal on Selected Areas in Communications, vol. 31, no. 2, pp. 264-273, 2013.

[27] C. Masouros, M. Sellathurai, and T. Ratnarajah, "Large-scale MIMO transmitters in fixed physical spaces: the effect of transmit correlation and mutual coupling," IEEE Transactions on Communications, vol. 61, no. 7, pp. 2794-2804, 2013.

[28] B. Clerckx, C. Craeye, D. Vanhoenacker-Janvier, and C. Oestges, "Impact of antenna coupling on $2 \times 2$ MIMO communications," IEEE Transactions on Vehicular Technology, vol. 56, no. 3, pp. 1009-1018, 2007.

[29] C. Masouros, J. Chen, K. Tong, M. Sellathurai, and T. Ratnarajah, "Towards massive-MIMO transmitters: on the effects of deploying increasing antennas in fixed physical space," in Proceedings of the Future Network and Mobile Summit, pp. 1-10, 2013.

[30] C. A. Balanis, Antenna Theory: Analysis and Design, John Wiley \& Sons, Hoboken, NJ, USA, 2012.

[31] X. Gao, F. Tufvesson, O. Edfors, and F. Rusek, "Channel behavior for very-large MIMO systems-initial characterization," in Proceedings of the Joint Workshop on Small Cell Cooperative Communications (COST IC1004 '12), 2012.

[32] E. H. Aggoune, "A non-stationary wideband channel model for large MIMO communication systems".
[33] A. Ghazal, C.-X. Wang, H. Haas et al., "A non-stationary MIMO channel model for high-speed train communication systems," in Proceedings of the 75th IEEE Vehicular Technology Conference (VTC '12), pp. 1-5, Yokohama, Japan, June 2012.

[34] T. Aulin, "A modified model for the fading signal at a mobile radio channel," IEEE Transactions on Vehicular Technology, vol. 28, no. 3, pp. 182-203, 1979.

[35] K. Kalliola, H. Laitinen, P. Vainikainen, M. Toeltsch, J. Laurila, and E. Bonek, "3-D double-directional radio channel characterization for urban macrocellular applications," IEEE Transactions on Antennas and Propagation, vol. 51, no. 11, pp. 3122-3133, 2003.

[36] J. Meinilä, P. Kyösti, L. Hentilä et al., "D5.3: WINNER+ final channel models," Wireless World Initiative New Radio WINNER, 2010.

[37] “WINNER II interim channel models," IST-WINNER, 2006. 

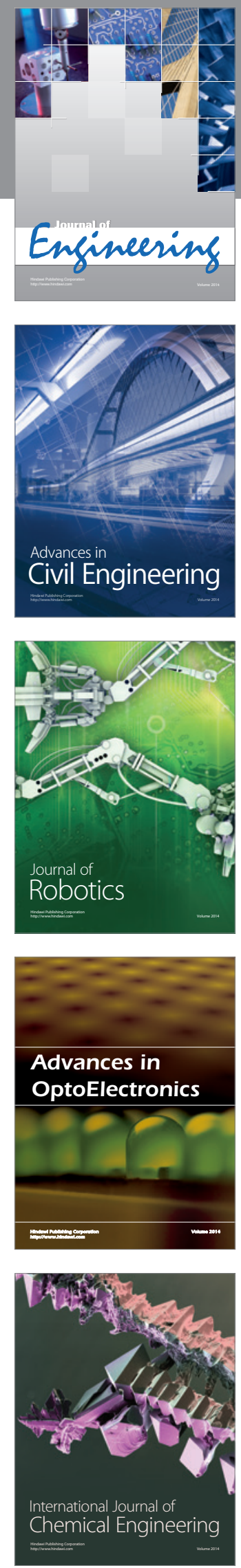

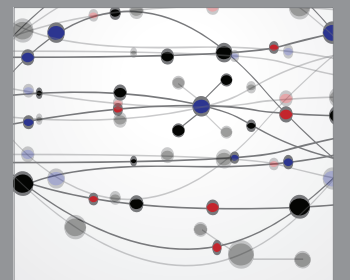

The Scientific World Journal
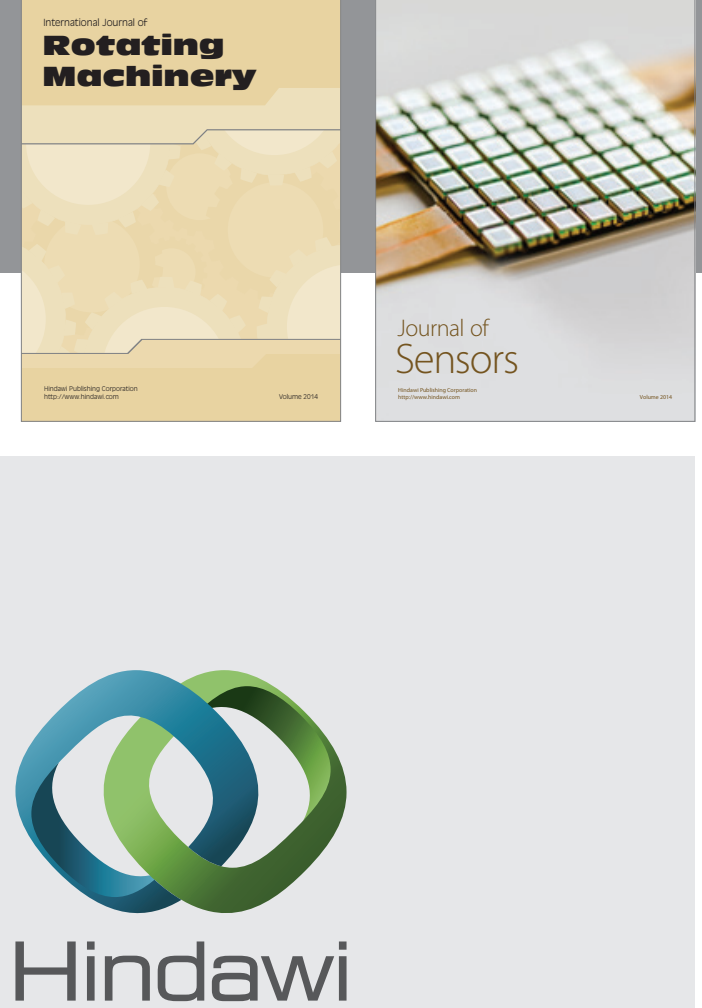

Submit your manuscripts at http://www.hindawi.com
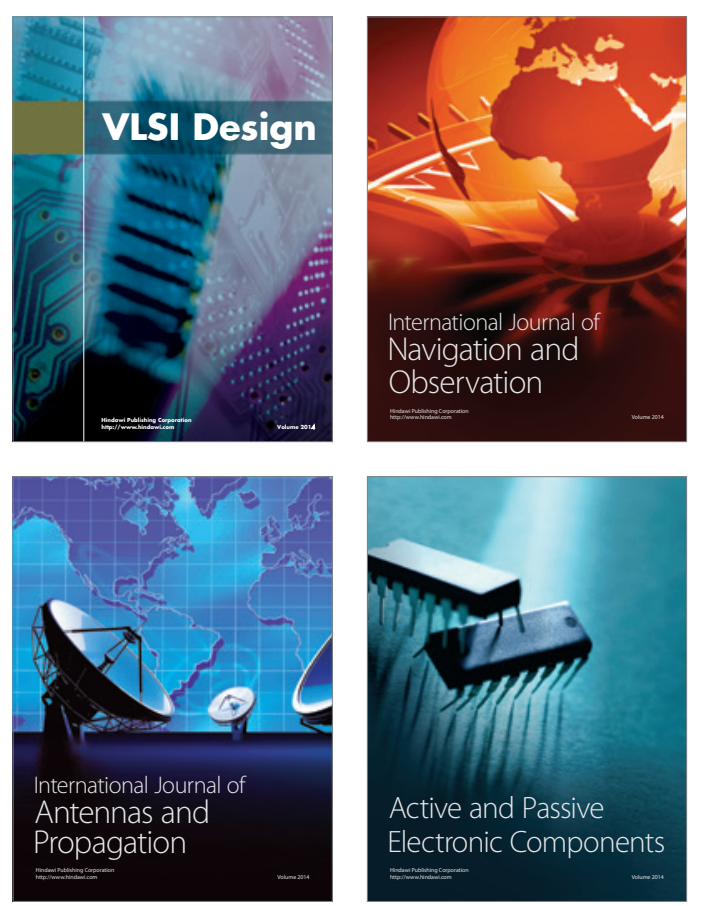
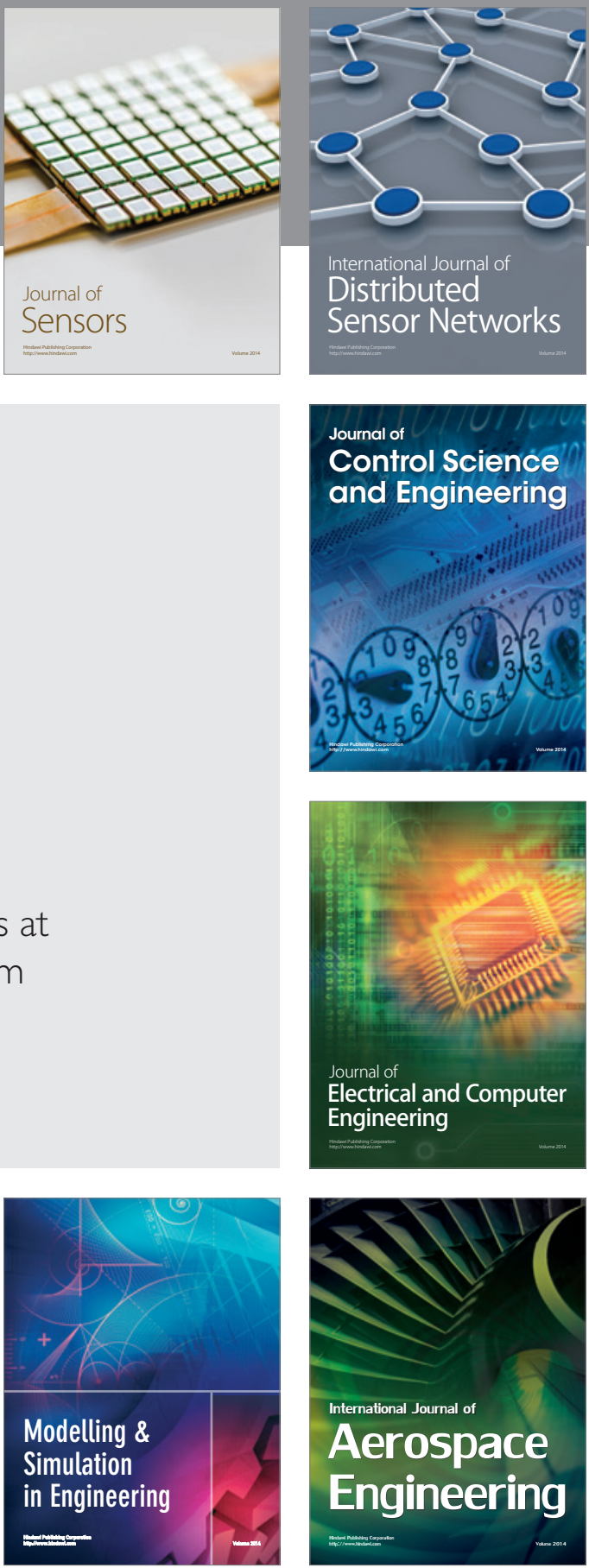

Journal of

Control Science

and Engineering
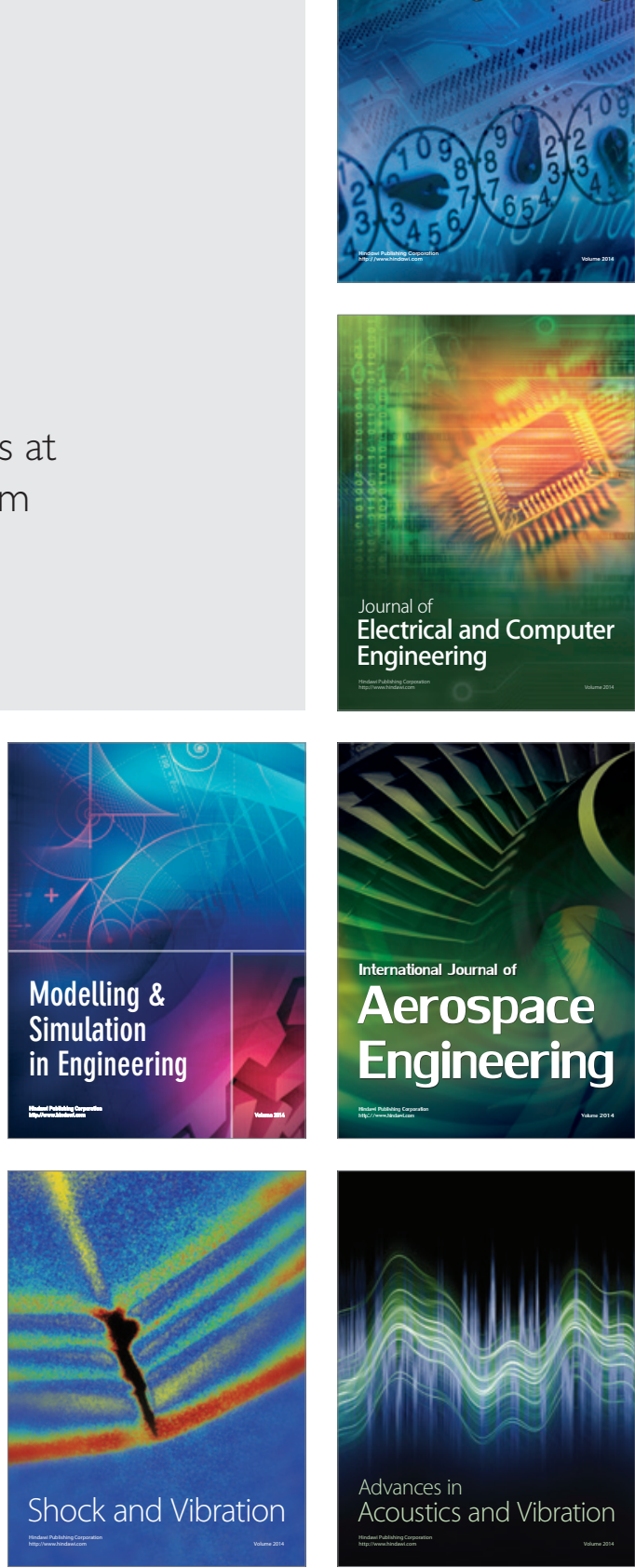